\title{
ITERATIVE APPROXIMATION OF SOLUTIONS OF NONLINEAR EQUATIONS OF HAMMERSTEIN TYPE
}

\author{
C. E. CHIDUME AND H. ZEGEYE
}

Received 27 October 2001

Suppose $X$ is a real $q$-uniformly smooth Banach space and $F, K: X \rightarrow X$ with $D(K)=F(X)=X$ are accretive maps. Under various continuity assumptions on $F$ and $K$ such that $0=u+K F u$ has a solution, iterative methods which converge strongly to such a solution are constructed. No invertibility assumption is imposed on $K$ and the operators $K$ and $F$ need not be defined on compact subsets of $X$. Our method of proof is of independent interest.

\section{Introduction}

Let $X$ be a real normed linear space with dual $X^{*}$. For $1<q<\infty$, we denote by $J_{q}$, the generalized duality mapping from $X$ to $2^{X^{*}}$ defined by

$$
J_{q}(x):=\left\{f^{*} \in X^{*}:\left\langle x, f^{*}\right\rangle=\|x\|\left\|f^{*}\right\|,\left\|f^{*}\right\|=\|x\|^{q-1}\right\},
$$

where $\langle\cdot, \cdot\rangle$ denotes the generalized duality pairing. If $q=2, J_{q}=J_{2}$ and is denoted by $J$. If $X^{*}$ is strictly convex, then $J_{q}$ is single-valued (see, e.g., [32]). A multivalued map $A$ with domain $D(A)$ in a normed linear space $X$ is said to be accretive if for every $x, y \in D(A)$, there exists $j_{q}(x-y) \in J_{q}(x-y)$ such that

$$
\left\langle\xi-\eta, j_{q}(x-y)\right\rangle \geq 0 \quad \text { for each } \xi \in A x, \eta \in A y .
$$

If $X$ is a Hilbert space, accretive operators are also called monotone. The accretive mappings were introduced independently in 1967 by Browder [6] and Kato [24]. Interest in such mappings stems mainly from their firm connection with equations of evolution. It is known (see, e.g., [33]) that many physically significant problems can be modelled by initial-value problems of the form

$$
x^{\prime}(t)+A x(t)=0, \quad x(0)=x_{0},
$$


where $A$ is an accretive operator in an appropriate Banach space. Typical examples, where such evolution equations occur, can be found in the heat, wave or Schrödinger equations. If in (1.3), $x(t)$ is independent of $t$, then (1.3) reduces to

$$
A u=0
$$

whose solutions correspond to the equilibrium points of system (1.3). Consequently, considerable research efforts have been devoted, especially within the past twenty years or so, to methods of finding approximate solutions (when they exist) of (1.4), and hence,

$$
u+A u=0
$$

One important generalization of (1.5) is the so-called equation of Hammerstein type (see, e.g., [22]) where a nonlinear integral equation of Hammerstein type is one of the form

$$
u(x)+\int_{\Omega} \kappa(x, y) f(y, u(y)) d y=h(x),
$$

where $d y$ is a $\sigma$-finite measure on the measure space $\Omega$. The real kernel $\kappa$ is defined on $\Omega \times \Omega, f$ is a real-valued function defined on $\Omega \times \mathfrak{R}$ and is, in general, nonlinear, and $h$ is a given function on $\Omega$. Now if we define an operator $K$ by

$$
K v(x):=\int_{\Omega} \kappa(x, y) v(y) d y, \quad x \in \Omega,
$$

and the so-called superposition or Nemytskii operator by $F u(y):=f(y, u(y))$, then the integral equation (1.6) can be put in operator theoretic form as follows:

$$
u+K F u=0,
$$

where, without loss of generality, we have taken $h \equiv 0$. Now it is obvious that equation $u+A u=0$ is a very special case of (1.8) in which $K=I$ (the identity operator on $X$ ) and $A:=F$. Interest in (1.8) stems mainly from the fact that several problems arising in differential equations, for instance, elliptic boundary value problems whose linear parts possess Greens functions can, as a rule, be transformed into form (1.8) (see, e.g., [27, Chapter IV]). Equations of Hammerstein type play a crucial role in the theory of optimal control systems (see, e.g., [21]). Several existence and uniqueness theorems have been proved for equations of the Hammerstein type (see, e.g., $[3,5,7,8,19,10])$.

For the iterative approximation of solutions of (1.4) and (1.5), the monotonicity/accretivity of $A$ is crucial. The Mann iteration scheme (see, e.g., [26]) and the Ishikawa iteration scheme (see, e.g., [23]) have successfully been employed (see, e.g., $[1,9,10,11,12,13,14,15,16,17,18,19,20,21,23,25,27,28,29,30$, $31,32,33,34])$. Attempts to apply these methods to (1.8) have not provided satisfactory results. In particular, the recursion formulas obtained involved $K^{-1}$ 
(see, e.g., $[12,15,28]$ ) and this is not convenient in applications. Part of the difficulty is the fact that the composition of two monotone operators need not be monotone. In the special case in which the operators are defined on subsets $D$ of $X$ which are compact (or more generally, angle-bounded), Brézis and Browder [2] have proved the strong convergence of a suitably defined Galerkin approximation to a solution of (1.8) (see also [4]).

It is our purpose in this paper to introduce a new method that contains an auxiliary operator, defined in an appropriate real Banach space in terms of $K$ and $F$, which under certain conditions, is accretive whenever $K$ and $F$ are, and whose zeros are solutions of (1.8). Moreover, the operators $K$ and $F$ need not be defined on compact or angle-bounded subset of $X$. Furthermore, our method which does not involve $K^{-1}$ provides an explicit algorithm for the computation of solutions of (1.8).

\section{Preliminaries}

Let $X$ be a real normed linear space of dimension $\geq 2$. The modulus of smoothness of $X$ is defined by

$$
\rho_{X}(\tau):=\sup \left\{\frac{\|x+y\|+\|x-y\|}{2}-1:\|x\|=1,\|y\|=\tau\right\}, \quad \tau>0 .
$$

If there exist a constant $c>0$ and a real number $1<q<\infty$, such that $\rho_{X}(\tau) \leq c \tau^{q}$, then $X$ is said to be $q$-uniformly smooth. Typical examples of such spaces are the Lebesgue $L_{p}$, the sequence $\ell_{p}$, and the Sobolev $W_{p}^{m}$ spaces for $1<p<\infty$ where

$$
L_{p}\left(\text { or } l_{p}\right) \text { or } W_{p}^{m}= \begin{cases}2 \text {-uniformly smooth } & \text { if } 2 \leq p<\infty \\ p \text {-uniformly smooth } & \text { if } 1<p<2\end{cases}
$$

A Banach space $X$ is called uniformly smooth if $\lim _{\tau \rightarrow 0} \rho_{X}(\tau) / \tau=0$. A multivalued map $A$ is said to be $m$-accretive if it is accretive and $R(I+\lambda A$ ) (range of $(I+\lambda A))=X$, for all $\lambda>0$, where $I$ is the identity mapping. $A$ is said to be $\phi$ strongly accretive if for every $x, y \in D(A)$, there exist $j_{q}(x-y) \in J_{q}(x-y)$ and a strictly increasing function $\phi:[0, \infty) \rightarrow[0, \infty), \phi(0)=0$ such that

$$
\left\langle\xi-\eta, j_{q}(x-y)\right\rangle \geq \phi(\|x-y\|)\|x-y\|^{q-1},
$$

for each $\xi \in A x, \eta \in A y$, and it is strongly accretive if for each $x, y \in D(A)$, there exist $j_{q}(x-y) \in J_{q}(x-y)$ and a constant $k \in(0,1)$ such that

$$
\left\langle\xi-\eta, j_{q}(x-y)\right\rangle \geq k\|x-y\|^{q} \quad \text { for each } \xi \in A x, \eta \in A y .
$$

Let $\mathrm{CB}(X)$ be a family of all nonempty closed bounded subsets of $X$. A multivalued mapping $A: X \rightarrow \mathrm{CB}(X)$ is said to be uniformly continuous if for every given $\varepsilon>0$, there exists a $\delta>0$ such that for any given $x, y \in X$ with $\|x-y\|<\delta$, we 
have $H(A x, A y)<\varepsilon$ where $H$ is the Hausdorff metric on $\mathrm{CB}(X)$, that is, for any given $D, F \in \mathrm{CB}(X)$,

$$
H(D, F):=\max \left\{\sup _{x \in D} \inf d(x, y), \inf _{x \in D} \sup _{y \in F} d(x, y)\right\} .
$$

In the sequel, we will need the following results.

Theorem 2.1 [32]. Let $q>1$ and $X$ be a real Banach space. Then the following are equivalent:

(1) $X$ is q-uniformly smooth;

(2) there exists a constant $d_{q}>0$ such that for all $x, y \in X$

$$
\|x+y\|^{q} \leq\|x\|^{q}+q\left\langle y, j_{q}(x)\right\rangle+d_{q}\|y\|^{q}
$$

(3) there exists a constant $c_{q}>0$ such that for all $x, y \in X$ and $\lambda \in[0,1]$

$$
\|(1-\lambda) x+\lambda y\|^{q} \geq(1-\lambda)\|x\|^{q}+\lambda\|y\|^{q}-w_{q}(\lambda) c_{q}\|x-y\|^{q},
$$

where $w_{q}(\lambda)=\lambda^{q}(1-\lambda)+\lambda(1-\lambda)^{q}$.

Theorem 2.2 [17]. Let $X$ be a real uniformly smooth Banach space. Let $A: X \rightarrow X$ be a bounded $\phi$-strongly accretive map. Assume $0=A x$ has a solution $x^{*} \in X$. Then, there exists a real number $\gamma_{0}>0$ such that if the real sequence $\left\{\alpha_{n}\right\} \subset\left[0, \gamma_{0}\right]$ satisfies the following conditions: (i) $\lim \alpha_{n}=0$; (ii) $\sum \alpha_{n}=\infty$, then for arbitrary $x_{0} \in X$ the sequence $\left\{x_{n}\right\}$, defined by

$$
x_{n+1}:=x_{n}-\alpha_{n} A x_{n}, \quad n \geq 0,
$$

converges strongly to $x^{*}$, the unique solution of $A x=0$.

Theorem 2.3 [11]. Let $X$ be an arbitrary real Banach space. Let $A: X \rightarrow X$ be a Lipschitz and strongly accretive map with Lipschitz constant $L>0$ and strong accretivity constant $\lambda \in(0,1)$. Assume that $A x=0$ has a solution $x^{*} \in X$. Define $A_{\varepsilon}: X \rightarrow X$ by $A_{\varepsilon} x:=x-\varepsilon A x$ for $x \in X$ where $\varepsilon:=1 / 2\{\lambda /(1+L(3+L-\lambda))\}$. For arbitrary $x_{0} \in X$, define the Picard sequence $\left\{x_{n}\right\}$ in $X$ by $x_{n+1}=A_{\varepsilon} x_{n}, n \geq 0$. Then, $\left\{x_{n}\right\}$ converges strongly to $x^{*}$ with $\left\|x_{n+1}-x^{*}\right\| \leq \delta^{n}\left\|x_{1}-x^{*}\right\|$ where $\delta:=$ $(1-1 / 2 \lambda \varepsilon) \in(0,1)$. Moreover, $x^{*}$ is unique.

\section{Main results}

Lemma 3.1. For $q>1$, let $X$ be a real $q$-uniformly smooth Banach space. Let $E:=$ $X \times X$ with norm

$$
\|z\|_{E}:=\left(\|u\|_{X}^{q}+\|v\|_{X}^{q}\right)^{1 / q}
$$

for arbitrary $z=[u, v] \in E$. Let $E^{*}:=X^{*} \times X^{*}$ denote the dual space of $E$. For 
arbitrary $x=\left[x_{1}, x_{2}\right] \in E$, define the map $j_{q}^{E}: E \rightarrow E^{*}$ by $j_{q}^{E}(x)=j_{q}^{E}\left[x_{1}, x_{2}\right]:=$ $\left[j_{q}^{X}\left(x_{1}\right), j_{q}^{X}\left(x_{2}\right)\right]$, so that for arbitrary $z_{1}=\left[u_{1}, v_{1}\right], z_{2}=\left[u_{2}, v_{2}\right]$ in $E$ the duality pairing $\langle\cdot, \cdot\rangle$ is given by

$$
\left\langle z_{1}, j_{q}^{E}\left(z_{2}\right)\right\rangle=\left\langle u_{1}, j_{q}^{X}\left(u_{2}\right)\right\rangle+\left\langle v_{1}, j_{q}^{X}\left(v_{2}\right)\right\rangle .
$$

Then,

(a) $E$ is q-uniformly smooth;

(b) $j_{q}^{E}$ is a single-valued duality mapping on $E$.

Proof. (a) Let $x=\left[x_{1}, x_{2}\right], y=\left[y_{1}, y_{2}\right]$ be arbitrary elements of $E$. It suffices to show that $x$ and $y$ satisfy condition (2) of Theorem 2.1. We compute as follows:

$$
\begin{aligned}
\|x+y\|_{E}^{q}= & \left\|\left[x_{1}+y_{1}, x_{2}+y_{2}\right]\right\|_{E}^{q}=\left\|x_{1}+y_{1}\right\|_{X}^{q}+\left\|x_{2}+y_{2}\right\|_{X}^{q} \\
\leq & \left\|x_{1}\right\|_{X}^{q}+\left\|x_{2}\right\|_{X}^{q}+d_{q}\left(\left\|y_{1}\right\|_{X}^{q}+\left\|y_{2}\right\|_{X}^{q}\right) \\
& +q\left\{\left\langle y_{1}, j_{q}^{X}\left(x_{1}\right)\right\rangle+\left\langle y_{2}, j_{q}^{X}\left(x_{2}\right)\right\rangle\right\}
\end{aligned}
$$

for some constants $d_{q}>0$ (using (2) of Theorem 2.1 since $X$ is $q$-uniformly smooth). It follows that

$$
\|x+y\|_{E}^{q} \leq\|x\|_{E}^{q}+q\left\langle y, j_{q}^{E}(x)\right\rangle+d_{q}\|y\|_{E}^{q}
$$

So, the result follows from Theorem 2.1. Since $E$ is $q$-uniformly smooth, it is smooth and so any duality mapping on $E$ is single-valued.

(b) For arbitrary $x=\left[x_{1}, x_{2}\right] \in E$, let $j_{q}^{E}(x)=j_{q}^{E}\left[x_{1}, x_{2}\right]=\psi_{q}$. Then $\psi_{q}=$ $\left[j_{q}^{X}\left(x_{1}\right), j_{q}^{X}\left(x_{2}\right)\right]$ in $E^{*}$. Observe that for $p>1$ such that $1 / p+1 / q=1$,

$$
\begin{aligned}
\left\|\psi_{q}\right\|_{E^{*}} & =\left(\left\|\left[j_{q}^{X}\left(x_{1}\right), j_{q}^{X}\left(x_{2}\right)\right]\right\|\right)^{1 / p}=\left(\left\|j_{q}\left(x_{1}\right)\right\|_{X^{*}}^{p}+\left\|j_{q}\left(x_{2}\right)\right\|_{X^{*}}^{p}\right)^{1 / p} \\
& =\left(\left\|x_{1}\right\|_{X}^{(q-1) p}+\left\|x_{2}\right\|_{X}^{(q-1) p}\right)^{1 / p}=\left(\left\|x_{1}\right\|_{X}^{q}+\left\|x_{2}\right\|_{X}^{q}\right)^{(q-1) / q} \\
& =\|x\|_{X}^{q-1} .
\end{aligned}
$$

Hence, $\left\|\psi_{q}\right\|_{E^{*}}=\|x\|_{E}^{q-1}$. Furthermore,

$$
\begin{aligned}
\left\langle x, \psi_{q}\right\rangle & =\left\langle\left[x_{1}, x_{2}\right],\left[j_{q}^{X}\left(x_{1}\right), j_{q}^{X}\left(x_{2}\right)\right]\right\rangle=\left\langle x_{1}, j_{q}^{X}\left(x_{1}\right)\right\rangle+\left\langle x_{2}, j_{q}^{X}\left(x_{2}\right)\right\rangle \\
& =\left\|x_{1}\right\|_{X}^{q}+\left\|x_{2}\right\|_{X}^{q}=\left(\left\|x_{1}\right\|_{X}^{q}+\left\|x_{2}\right\|_{X}^{q}\right)^{1 / q}\left(\left\|x_{1}\right\|_{X}^{q}+\left\|x_{2}\right\|_{X}^{q}\right)^{(q-1) / q} \\
& =\|x\|_{E} \cdot\|\psi\|_{E^{*}}^{q-1} .
\end{aligned}
$$

Hence, $j_{q}^{E}$ is a single-valued duality mapping on $E$.

Lemma 3.2. Let $X$ be a real $q$-uniformly smooth Banach space. Let $F, K: X \rightarrow X$ be maps with $D(K)=F(X)=X$ such that the following conditions hold: 
(i) for each $u_{1}, u_{2} \in D(F)$, there exists a strictly increasing function $\phi_{1}:[0, \infty)$ $\rightarrow[0, \infty), \phi_{1}(0)=0$ such that

$$
\left\langle F u_{1}-F u_{2}, j_{q}\left(u_{1}-u_{2}\right)\right\rangle \geq \phi_{1}\left(\left\|u_{1}-u_{2}\right\|\right)\left\|u_{1}-u_{2}\right\|^{q-1}
$$

(ii) for each $u_{1}, u_{2} \in D(K)$, there exists a strictly increasing function $\phi_{2}:[0, \infty)$ $\rightarrow[0, \infty), \phi_{2}(0)=0$ such that

$$
\left\langle K u_{1}-K u_{2}, j_{q}\left(u_{1}-u_{2}\right)\right\rangle \geq \phi_{2}\left(\left\|u_{1}-u_{2}\right\|\right)\left\|u_{1}-u_{2}\right\|^{q-1}
$$

(iii) $\phi_{i}(t) \geq\left(d+r_{i}\right) t$ for all $t \in[0, \infty)$ and for some $r_{i}>0, i=1,2$ where $d:=$ $q^{-1}\left(1+d_{q}-c^{-1} 2^{q-1}\right) ; c=\max \left\{1, c_{q}\right\}$ and $d_{q}, c_{q}$ are the constants appearing in inequalities (2.6) and (2.7), respectively.

Let $E:=X \times X$ with norm $\|z\|_{E}^{q}=\|u\|_{X}^{q}+\|v\|_{X}^{q}$ for $z=(u, v) \in E$ and define $a$ map $T: E \rightarrow 2^{E}$ by $T z:=T(u, v)=(F u-v, u+K v)$. Then for each $z_{1}, z_{2} \in E$, there exists a strictly increasing function $\phi:[0, \infty) \rightarrow[0, \infty)$ with $\phi(0)=0$ such that

$$
\left\langle T z_{1}-T z_{2}, j_{q}^{E}\left(z_{1}-z_{2}\right)\right\rangle \geq \phi\left(\left\|z_{1}-z_{2}\right\|\right)\left\|z_{1}-z_{2}\right\|^{q-1} .
$$

Proof. Define $\phi:[0, \infty) \rightarrow[0, \infty)$ by $\phi(t):=\min \left\{r_{1}, r_{2}\right\} t$ for each $t \in[0, \infty)$. Observe that $\phi$ is a strictly increasing function with $\phi(0)=0$. Furthermore, for $q>$ $1, z_{1}=\left(u_{1}, v_{1}\right)$ and $z_{2}=\left(u_{2}, v_{2}\right)$ arbitrary elements in $E$, we have $\left\langle z_{1}, j_{q}^{E}\left(z_{2}\right)\right\rangle=$ $\left\langle u_{1}, j_{q}\left(u_{2}\right)\right\rangle+\left\langle v_{1}, j_{q}\left(v_{2}\right)\right\rangle$. Thus, we have the following estimates:

$$
\begin{aligned}
\left\langle T z_{1}-T z_{2},\right. & \left.j_{q}^{E}\left(z_{1}-z_{2}\right)\right\rangle \\
= & \left\langle F u_{1}-F u_{2}-\left(v_{1}-v_{2}\right), j_{q}\left(u_{1}-u_{2}\right)\right\rangle \\
& +\left\langle K v_{1}-K v_{2}+\left(u_{1}-u_{2}\right), j_{q}\left(v_{1}-v_{2}\right)\right\rangle \\
= & \left\langle F u_{1}-F u_{2}, j_{q}\left(u_{1}-u_{2}\right)\right\rangle-\left\langle v_{1}-v_{2}, j_{q}\left(u_{1}-u_{2}\right)\right\rangle \\
& +\left\langle K v_{1}-K v_{2}, j_{q}\left(v_{1}-v_{2}\right)\right\rangle+\left\langle u_{1}-u_{2}, j_{q}\left(v_{1}-v_{2}\right)\right\rangle \\
\geq & \phi_{1}\left(\| u_{1}-u_{2}||\right)\left\|u_{1}-u_{2}\right\|^{q-1}+\phi_{2}\left(\left\|v_{1}-v_{2}\right\|\right)\left\|v_{1}-v_{2}\right\|^{q-1} \\
& -\left\langle v_{1}-v_{2}, j_{q}\left(u_{1}-u_{2}\right)\right\rangle+\left\langle u_{1}-u_{2}, j_{q}\left(v_{1}-v_{2}\right)\right\rangle .
\end{aligned}
$$

Since $X$ is real $q$-uniformly smooth, inequality (2.7) holds for each $x, y \in X$. Setting $\lambda=1 / 2$ in this inequality yields the following estimate:

$$
\|x+y\|^{q}+\|x-y\|^{q} \geq c^{-1} 2^{q-1}\left(\|x\|^{q}+\|y\|^{q}\right),
$$

where $c=\max \left\{1, c_{q}\right\}$. Furthermore, from inequality (2.6), replacing $y$ by $-y$, we obtain the following inequality:

$$
-\left\langle y, j_{q}(x)\right\rangle \geq q^{-1}\left(\|x-y\|^{q}-\|x\|^{q}-d_{q}\|y\|^{q}\right) .
$$


Using (3.10), (3.12), (2.6), and (3.11), we obtain the following estimates:

$$
\begin{aligned}
\left\langle T z_{1}-\right. & \left.T z_{2}, j_{q}^{E}\left(z_{1}-z_{2}\right)\right\rangle \\
\geq & \phi_{1}\left(\left\|u_{1}-u_{2}\right\|\right)\left\|u_{1}-u_{2}\right\|^{q-1}+\phi_{2}\left(\left\|v_{1}-v_{2}\right\|\right)\left\|v_{1}-v_{2}\right\|^{q-1} \\
& +q^{-1}\left(\left\|v_{1}-v_{2}-\left(u_{1}-u_{2}\right)\right\|^{q}-\left\|u_{1}-u_{2}\right\|^{q}-d_{q}\left\|v_{1}-v_{2}\right\|^{q}\right) \\
& +q^{-1}\left(\left\|v_{1}-v_{2}+\left(u_{1}-u_{2}\right)\right\|^{q}-\left\|v_{1}-v_{2}\right\|^{q}-d_{q}\left\|u_{1}-u_{2}\right\|^{q}\right) \\
\geq & \phi_{1}\left(\left\|u_{1}-u_{2}\right\|\right)\left\|u_{1}-u_{2}\right\|^{q-1}+\phi_{2}\left(\left\|v_{1}-v_{2}\right\|\right)\left\|v_{1}-v_{2}\right\|^{q-1} \\
& +q^{-1} c^{-1} 2^{q-1}\left(\left\|u_{1}-u_{2}\right\|^{q}+\left\|v_{1}-v_{2}\right\|^{q}\right) \\
& -q^{-1}\left\{\left(1+d_{q}\right)\left\|u_{1}-u_{2}\right\|^{q}+\left(1+d_{q}\right)\left\|v_{1}-v_{2}\right\|^{q}\right\} \\
\geq & \left\{\phi_{1}\left(\left\|u_{1}-u_{2}\right\|\right)-d\left\|u_{1}-u_{2}\right\|\right\}\left\|u_{1}-u_{2}\right\|^{q-1} \\
& +\left\{\phi_{2}\left(\left\|v_{1}-v_{2}\right\|\right)-d\left\|v_{1}-v_{2}\right\|\right\}\left\|v_{1}-v_{2}\right\|^{q-1} \\
\geq & \min \left\{r_{1}, r_{2}\right\}\left\{\left\|u_{1}-u_{2}\right\|^{q}+\left\|v_{1}-v_{2}\right\|^{q}\right\} \\
= & \min \left\{r_{1}, r_{2}\right\}\left\|z_{1}-z_{2}\right\| \cdot\left\|z_{1}-z_{2}\right\|^{q-1} \\
= & \phi\left(\left\|z_{1}-z_{2}\right\|\right)\left\|z_{1}-z_{2}\right\|^{q-1},
\end{aligned}
$$

completing the proof of Lemma 3.2.

Corollary 3.3. Let $X$ be a real q-uniformly smooth Banach space. Let $F, K: X \rightarrow$ $X$ be maps with $D(K)=F(X)=X$ such that the following conditions hold:

(i) for each $u_{1}, u_{2} \in D(F)$, there exists $\alpha>0$ such that

$$
\left\langle F u_{1}-F u_{2}, j_{q}\left(u_{1}-u_{2}\right)\right\rangle \geq \alpha\left\|u_{1}-u_{2}\right\|^{q} ;
$$

(ii) for each $u_{1}, u_{2} \in D(K)$, there exists $\beta>0$ such that

$$
\left\langle K u_{1}-K u_{2}, j_{q}\left(u_{1}-u_{2}\right)\right\rangle \geq \beta\left\|u_{1}-u_{2}\right\|^{q}
$$

(iii) $\alpha, \beta>d:=q^{-1}\left(1+d_{q}-c^{-1} 2^{q-1}\right)$ and $\gamma:=\min \{\alpha-d, \beta-d\}$ where $c$ and $d_{q}$ are as in (3.11) and (2.6), respectively.

Let $E$ and $T$ be defined as in Lemma 3.2. Then, for $z_{1}, z_{2} \in E$, we have that

$$
\left\langle T z_{1}-T z_{2}, j_{q}^{E}\left(z_{1}-z_{2}\right)\right\rangle \geq \gamma\left\|z_{1}-z_{2}\right\|^{q} .
$$

Proof. Let $\alpha, \beta$, and $\gamma$ be real constants satisfying (iii), then following precisely the method of proof of Lemma 3.2, we get the required result.

Corollary 3.4. Let $X=H$ be a real Hilbert space. Let $F, K: H \rightarrow H$ be maps with $D(K)=F(X)=X$ such that conditions (i) and (ii) of Corollary 3.3 are satisfied. Let $\alpha, \beta>0, E$, and $T$ be defined as in Corollary 3.3. Then, for $z_{1}, z_{2} \in E$, we have 
that

$$
\left\langle T z_{1}-T z_{2}, j_{q}^{E}\left(z_{1}-z_{2}\right)\right\rangle \geq \gamma\left\|z_{1}-z_{2}\right\|^{q},
$$

where $\gamma:=\min \{\alpha, \beta\}$.

Proof. Since, for Hilbert spaces, the duality mapping $j_{q}^{E}$ is the identity map, $q=$ 2, $d_{q}=1, c=1$, the result follows from Corollary 3.3.

\subsection{Convergence theorems for Lipschitz maps}

Remark 3.5. If $K$ and $F$ are Lipschitzian maps with positive constants $L_{K}$ and $L_{F}$, respectively, then $T$ is Lipschitzian map with constant $L:=\left(d \max \left\{L_{F}^{q}+1\right.\right.$, $\left.\left.L_{K}^{q}+1\right\}\right)^{1 / q}$ for some constant $d>0$. Indeed, if $z_{1}=\left(u_{1}, v_{1}\right), z_{2}=\left(u_{2}, v_{2}\right)$ in $E$, then we have that

$$
\begin{aligned}
\left\|T z_{1}-T z_{2}\right\|^{q}= & \left\|\left(F u_{1}-F u_{2}\right)-\left(v_{1}-v_{2}\right)\right\|^{q}+\left\|u_{1}-u_{2}+K v_{1}-K v_{2}\right\|^{q} \\
\leq & {\left[L_{F}\left\|u_{1}-u_{2}\right\|+\left\|v_{1}-v_{2}\right\|\right]^{q}+\left[\left\|u_{1}-u_{2}\right\|+L_{K}\left\|v_{1}-v_{2}\right\|\right]^{q} } \\
\leq & d\left[L_{F}^{q}\left\|u_{1}-u_{2}\right\|^{q}+\left\|v_{1}-v_{2}\right\|^{q}+\left\|u_{1}-u_{2}\right\|^{q}+L_{K}^{q}\left\|v_{1}-v_{2}\right\|^{q}\right] \\
& \text { for some } d>0 \\
\leq & d \max \left\{L_{F}^{q}+1, L_{K}^{q}+1\right\}\left[\left\|u_{1}-u_{2}\right\|^{q}+\left\|v_{1}-v_{2}\right\|^{q}\right] \\
= & d \max \left\{L_{F}^{q}+1, L_{K}^{q}+1\right\}\left\|z_{1}-z_{2}\right\|^{q} .
\end{aligned}
$$

Thus, $\left\|T z_{1}-T z_{2}\right\| \leq L\left\|z_{1}-z_{2}\right\|$.

Consequently, we have the following theorem.

Theorem 3.6. Let $X$ be real q-uniformly smooth Banach space. Let $F, K: X \rightarrow X$ be Lipschitzian maps with positive constants $L_{K}$ and $L_{F}$, respectively such that $D(K)=$ $F(X)=X$ with the following conditions:

(i) there exists $\alpha>0$ such that

$$
\left\langle F u_{1}-F u_{2}, j_{q}\left(u_{1}-u_{2}\right)\right\rangle \geq \alpha\left\|u_{1}-u_{2}\right\|^{q}, \quad \forall u_{1}, u_{2} \in D(F) ;
$$

(ii) there exists $\beta>0$ such that

$$
\left\langle K u_{1}-K u_{2}, j_{q}\left(u_{1}-u_{2}\right)\right\rangle \geq \beta\left\|u_{1}-u_{2}\right\|^{q}, \quad \forall u_{1}, u_{2} \in D(K)
$$

(iii) $\alpha, \beta>d:=q^{-1}\left(1+d_{q}-c^{-1} 2^{q-1}\right)$ and $\gamma:=\min \{\alpha-d, \beta-d\}$.

Assume that $u+K F u=0$ has solution $u^{*}$, let $E:=X \times X$ be with norm $\|z\|_{E}^{q}=$ $\|u\|_{X}^{q}+\|v\|_{X}^{q}$ for $z=(u, v) \in E$, and define the map $T: E \rightarrow E$ by $T z:=T(u, v)=$ $(F u-v, K v+u)$. Let $L$ be Lipschitz constant of $T$ and $\varepsilon:=(1 / 2)(\gamma /(1+$ $L(3+L-\gamma)))$. Define the map $A_{\varepsilon}: E \rightarrow E$ by $A_{\varepsilon} z:=z-\varepsilon T z$ for each $z \in E$. For arbitrary $z_{0} \in E$, define the Picard sequence $\left\{z_{n}\right\}$ in $E$ by $z_{n+1}:=A_{\varepsilon} z_{n}, n \geq 0$. Then 
$\left\{z_{n}\right\}$ converges strongly to $z^{*}=\left[u^{*}, v^{*}\right]$, the unique solution of the equation $T z=0$ with $\left\|z_{n+1}-z^{*}\right\| \leq \delta^{n}\left\|z_{1}-z^{*}\right\|$ where $v^{*}=F u^{*}$ and $u^{*}$ is the solution of the equation $u+K F u=0$ and $\delta:=(1-(1 / 2) \gamma \varepsilon) \in(0,1)$.

Proof. Observe that $u^{*}$ is a solution of $u+K F u=0$ if and only if $z^{*}=\left[u^{*}, v^{*}\right]$ is a solution of $T z=0$ for $v^{*}=F u^{*}$. Hence, $T z=0$ has a solution $z^{*}=\left[u^{*}, v^{*}\right]$ in $E$. Since $T$ is Lipschitz, and by Corollary 3.3, it is strongly accretive with constant $\gamma$ (which, without loss of generality we may assume, is in $(0,1))$. The conclusion follows from Theorem 2.3.

Remark 3.7. Since $L^{p}$ spaces, $1<p<\infty$, are $q$-uniformly smooth spaces where $q=\min \{2, p\}$, then $c_{q}=d_{q} \geq 1$ and is given by

$$
c_{q}=d_{q}= \begin{cases}\frac{1+b^{q-1}}{(1+b)^{q-1}}, & \text { if } 1<p<2, \\ p-1, & \text { if } 2 \leq p<\infty\end{cases}
$$

where $b$ is the unique solution of the equation $(q-2) t^{q-1}+(q-1) t^{q-2}-1=0$, $0<t<1$ (see, e.g., [32]).

As a consequence of Theorem 3.6 and Remark 3.7, we have the following corollaries.

Corollary 3.8. Suppose $X=L_{p}(1<p<\infty)$. Let $F, K: X \rightarrow X$ be Lipschitzian maps with positive constants $L_{K}$ and $L_{F}$, respectively, and $D(K)=F(X)=X$ with conditions (i) and (ii) of Theorem 3.6. Suppose $\alpha, \beta>d$ and $\gamma:=\min \{\alpha-d, \beta-$ d) where

$$
d:= \begin{cases}\frac{1}{2}\left(p-\frac{2}{p-1}\right), & \text { if } 2 \leq p<\infty, \\ q^{-1}\left(1+\frac{1+b^{q-1}}{(1+b)^{q-1}}-\frac{(1+b)^{q-1}}{1+b^{q-1}} 2^{q-1}\right), & \text { if } 1<p<2 .\end{cases}
$$

Assume that $u+K F u=0$ has solution $u^{*}$ and set $E$ and $T$ as in Theorem 3.6. Let $L, \varepsilon, A_{\varepsilon}$, and $\left\{z_{n}\right\}$ be defined as in Theorem 3.6. Then $\left\{z_{n}\right\}$ converges strongly to $z^{*}=\left[u^{*}, v^{*}\right]$ with $\left\|z_{n+1}-z^{*}\right\| \leq \delta^{n}\left\|z_{1}-z^{*}\right\|$ where $\delta:=(1-(1 / 2) \gamma \varepsilon) \in(0,1)$, $v^{*}=F u^{*}$ and $u^{*}$ is the unique solution of $u+K F u=0$.

Corollary 3.9. Let $X=H$ be a real Hilbert space. Let $F$ and $K$ be as in Corollary 3.8. Suppose $\alpha, \beta>0$ and $\gamma:=\min \{\alpha, \beta\}$. Assume that $u+K F u=0$ has solution $u^{*}$ and set $E$ and $T$ as in Corollary 3.8. Let $L, \varepsilon, A_{\varepsilon}$, and $\left\{z_{n}\right\}$ be defined as in Corollary 3.8. Then $\left\{z_{n}\right\}$ converges strongly to $z^{*}=\left[u^{*}, v^{*}\right]$ with $\left\|z_{n+1}-z^{*}\right\| \leq$ $\delta^{n}\left\|z_{1}-z^{*}\right\|$ where $\delta:=(1-(1 / 2) \gamma \varepsilon) \in(0,1), v^{*}=F u^{*}$ and $u^{*}$ is the unique solution of $u+K F u=0$.

Proof. The proof follows from Corollary 3.8 with $p=2$. 


\subsection{Convergence theorems for bounded maps}

Theorem 3.10. Let $X$ be a real q-uniformly smooth Banach space. Let $F, K: X \rightarrow X$ with $D(K)=F(X)=X$ be bounded maps such that the following conditions hold:

(i) for each $u_{1}, u_{2} \in X$, there exists a strictly increasing function $\phi_{1}:[0, \infty) \rightarrow$ $[0, \infty), \phi_{1}(0)=0$ such that

$$
\left\langle F u_{1}-F u_{2}, j_{q}\left(u_{1}-u_{2}\right)\right\rangle \geq \phi_{1}\left(\left\|u_{1}-u_{2}\right\|\right)\left\|u_{1}-u_{2}\right\|^{q-1} ;
$$

(ii) for each $u_{1}, u_{2} \in X$, there exists a strictly increasing function $\phi_{2}:[0, \infty) \rightarrow$ $[0, \infty), \phi_{2}(0)=0$ such that

$$
\left\langle K u_{1}-K u_{2}, j_{q}\left(u_{1}-u_{2}\right)\right\rangle \geq \phi_{2}\left(\left\|u_{1}-u_{2}\right\|\right)\left\|u_{1}-u_{2}\right\|^{q-1}
$$

(iii) $\phi_{i}(t) \geq\left(d+r_{i}\right) t$ for all $t \in[0, \infty)$ and $i=1,2$ for some $r_{i}>0$ and $d$ is as in Lemma 3.2.

Assume that $0=u+K F u$ has solution $u^{*}$ in $X$. Let $E:=X \times X$ be with norm $\|z\|_{E}^{q}=\|u\|_{X}^{q}+\|v\|_{X}^{q}$ for $z=(u, v) \in E$ and define the map $T: E \rightarrow E$ by $T z:=$ $T(u, v)=(F u-v, u+K v)$. Then there exists a real number $\gamma_{0}>0$ such that, if the real sequence $\left\{\alpha_{n}\right\} \subset\left[0, \gamma_{0}\right]$ satisfies the following conditions: (i) $\lim _{n \rightarrow \infty} \alpha_{n}=0$; (ii) $\sum \alpha_{n}=\infty$, then for arbitrary $z_{0} \in E$, the sequence $\left\{z_{n}\right\}$ defined by

$$
z_{n+1}:=z_{n}-\alpha_{n} T z_{n}, \quad n \geq 0,
$$

converges strongly to $z^{*}=\left[u^{*}, v^{*}\right]$ where $v^{*}=F u^{*}$ and $u^{*}$ is the unique solution of $0=u+K F u$.

Proof. Observe that since $K$ and $F$ are bounded maps, we have that $T$ is bounded map. Observe also that $u^{*}$ is the solution of $0=u+K F u$ in $X$ if and only if $z^{*}=$ $\left[u^{*}, v^{*}\right]$ is a solution of $0=T z$ in $E$ for $v^{*}=F u^{*}$. Thus, we obtain that $N(T)$ (null space of $T$ ) $\neq \varnothing$. Also by Lemma 3.2, $T$ is $\phi$-strongly accretive. Therefore, the conclusion follows from Theorem 2.2.

Following the method of proof of Theorem 3.10 and making use of Corollary 3.3, we obtain the following theorem.

Theorem 3.11. Let $X$ be a real q-uniformly smooth Banach space. Let $F, K: X \rightarrow X$ with $D(K)=F(X)=X$ be bounded maps such that the following conditions hold:

(i) for each $u_{1}, u_{2} \in D(F)$, there exists $\alpha>0$ such that

$$
\left\langle F u_{1}-F u_{2}, j_{q}\left(u_{1}-u_{2}\right)\right\rangle \geq \alpha\left\|u_{1}-u_{2}\right\|^{q} ;
$$

(ii) for each $u_{1}, u_{2} \in D(K)$, there exists $\beta>0$ such that

$$
\left\langle K u_{1}-K u_{2}, j_{q}\left(u_{1}-u_{2}\right)\right\rangle \geq \beta\left\|u_{1}-u_{2}\right\|^{q} ;
$$


(iii) $\alpha, \beta>d:=q^{-1}\left(1+d_{q}-c^{-1} 2^{q-1}\right)$ where $c$ and $d_{q}$ are as in (3.11) and (2.6), respectively.

Assume that $0=u+K F u$ has solution $u^{*}$. Let $E, T$, and $\left\{z_{n}\right\}$ be defined as in Theorem 3.10. Then, the conclusion of Theorem 3.10 holds.

Corollary 3.12. Let $X=L_{p}(1<p<\infty)$. Let $F, K: X \rightarrow X$ with $D(K)=F(X)=$ $X$ be bounded maps such that (i) and (ii) of Theorem 3.11 hold and $\alpha, \beta>d$ where $d$ is as in Corollary 3.8. Assume that $0=u+K F u$ has solution $u^{*}$. Let E, T, and $\left\{z_{n}\right\}$ be defined as in Theorem 3.11. Then the conclusion of Theorem 3.11 holds.

Proof. The proof follows from Theorem 3.11 with Remark 3.7.

Corollary 3.13. Let $X=H$ be real Hilbert space. Let $F$ and $K$ be as in Corollary 3.12 and $\alpha, \beta>0$. Assume that $0=u+K F u$ has solution $u^{*}$. Let $E, T$, and $\left\{z_{n}\right\}$ be defined as in Corollary 3.12. Then the conclusion of Corollary 3.12 holds.

Proof. The proof follows from Corollary 3.12 with $p=2$.

3.3. Explicit algorithms. The method of our proofs provides the following explicit algorithms for computing the solution of the equation $0=u+K F u$ in the space $X$.

(a) For Lipschitz operators (Theorem 3.6 and Corollaries 3.8, 3.9) with initial values $u_{0}, v_{0} \in X$, define the sequences $\left\{u_{n}\right\}$ and $\left\{v_{n}\right\}$ in $X$ as follows:

$$
\begin{aligned}
& u_{n+1}=u_{n}-\varepsilon\left(F\left(u_{n}\right)-v_{n}\right) ; \\
& v_{n+1}=v_{n}-\varepsilon\left(K\left(v_{n}\right)+u_{n}\right) .
\end{aligned}
$$

Then $u_{n} \rightarrow u^{*}$ in $X$, the unique solution $u^{*}$ of $0=u+K F u$ with $v^{*}=F u^{*}$ where $\varepsilon$ is as defined in Theorem 3.6.

(b) For bounded operators (Theorems 3.10, 3.11 and Corollaries 3.12, 3.13) with initial values $u_{0}, v_{0} \in X$, define the sequences $\left\{u_{n}\right\}$ and $\left\{v_{n}\right\}$ in $X$ as follows:

$$
\begin{aligned}
& u_{n+1}=u_{n}-\alpha_{n}\left(F u_{n}-v_{n}\right) \\
& v_{n+1}=v_{n}-\alpha_{n}\left(K v_{n}+u_{n}\right) .
\end{aligned}
$$

Then $u_{n} \rightarrow u^{*}$ in $X$, the unique solution $u^{*}$ of $0=u+K F u$ with $v^{*}=F u^{*}$ where $\alpha_{n}$ is as defined in Theorem 3.10.

\section{Acknowledgments}

The authors thank the referee for very useful comments which improved the presentation of this paper. The second author undertook this work while visiting the Abdus Salam International Center for Theoretical Physics, Trieste, Italy, as a postdoctoral fellow. 


\section{References}

[1] H. H. Bauschke, The approximation of fixed points of compositions of nonexpansive mappings in Hilbert space, J. Math. Anal. Appl. 202 (1996), no. 1, 150-159.

[2] H. Brézis and F. E. Browder, Some new results about Hammerstein equations, Bull. Amer. Math. Soc. 80 (1974), 567-572.

[3] Existence theorems for nonlinear integral equations of Hammerstein type, Bull. Amer. Math. Soc. 81 (1975), 73-78.

[4] Nonlinear integral equations and systems of Hammerstein type, Advances in Math. 18 (1975), no. 2, 115-147.

[5] F. E. Browder, Nonexpansive nonlinear operators in a Banach space, Proc. Nat. Acad. Sci. U.S.A. 54 (1965), 1041-1044.

[6] Nonlinear mappings of nonexpansive and accretive type in Banach spaces, Bull. Amer. Math. Soc. 73 (1967), 875-882.

[7] F. E. Browder, D. G. de Figueiredo, and C. P. Gupta, Maximal monotone operators and nonlinear integral equations of Hammerstein type, Bull. Amer. Math. Soc. 76 (1970), 700-705.

[8] F. E. Browder and C. P. Gupta, Monotone operators and nonlinear integral equations of Hammerstein type, Bull. Amer. Math. Soc. 75 (1969), 1347-1353.

[9] R. E. Bruck Jr., The iterative solution of the equation $y \in x+T x$ for a monotone operator $T$ in Hilbert space, Bull. Amer. Math. Soc. 79 (1973), 1258-1261.

[10] R. Sh. Chepanovich, Nonlinear Hammerstein equations and fixed points, Publ. Inst. Math. (Beograd) (N.S.) 35(49) (1984), 119-123.

[11] C. E. Chidume, Nonexpansive mappings, generalizations and iterative algorithms, Accepted to appear in Nonlinear Analysis and Applications, (R. P. Agarwal and D. O’Regan, Editors) Kluwer Academic Publishers (Research Monograph to appear in 2003).

[12] - Fixed point iterations for nonlinear Hammerstein equation involving nonexpansive and accretive mappings, Indian J. Pure Appl. Math. 20 (1989), no. 2, 129135.

[13] Iterative solutions of nonlinear equations of the strongly accretive type, Math. Nachr. 189 (1998), 49-60.

[14] C. E. Chidume and C. Moore, Fixed point iteration for pseudocontractive maps, Proc. Amer. Math. Soc. 127 (1999), no. 4, 1163-1170.

[15] C. E. Chidume and M. O. Osilike, Iterative solution for nonlinear integral equations of Hammerstein type, J. Nigerian Math. Soc. 11 (1992), no. 1, 9-18.

[16] C. E. Chidume and H. Zegeye, Global iterative schemes for accretive operators, J. Math. Anal. Appl. 257 (2001), no. 2, 364-377.

[17] C. E. Chidume, H. Zegeye, and B. Ntatin, A generalized steepest descent approximation for the zeros of m-accretive operators, J. Math. Anal. Appl. 236 (1999), no. 1, 4873.

[18] M. G. Crandall and A. Pazy, On the range of accretive operators, Israel J. Math. 27 (1977), no. 3-4, 235-246.

[19] D. G. de Figueiredo and C. P. Gupta, On the variational method for the existence of solutions of nonlinear equations of Hammerstein type, Proc. Amer. Math. Soc. 40 (1973), 470-476.

[20] L. Deng, On Chidume's open questions, J. Math. Anal. Appl. 174 (1993), no. 2, 441449 . 
[21] V. Dolezal, Monotone Operators and Applications in Control and Network Theory, Studies in Automation and Control, vol. 2, Elsevier Scientific Publishing, Amsterdam, 1979.

[22] A. Hammerstein, Nichtlineare Integralgleichungen nebst Anwendungen, Acta Math. 54 (1930), 117-176 (German).

[23] S. Ishikawa, Fixed points by a new iteration method, Proc. Amer. Math. Soc. 44 (1974), $147-150$.

[24] T. Kato, Nonlinear semigroups and evolution equations, J. Math. Soc. Japan 19 (1967), 508-520.

[25] Q. H. Liu, A convergence theorem for Ishikawa iterates of continuous generalized nonexpansive maps, J. Math. Anal. Appl. 165 (1992), no. 2, 305-309.

[26] W. R. Mann, Mean value methods in iteration, Proc. Amer. Math. Soc. 4 (1953), 506510.

[27] D. Pascali and S. Sburlan, Nonlinear Mappings of Monotone Type, Martinus Nijhoff Publishers, The Hague, 1978.

[28] S. Reich, Constructive techniques for accretive and monotone operators, Applied Nonlinear Analysis (Proc. Third Internat. Conf., Univ. Texas, Arlington, Tex, 1978), Academic Press, New York, 1979, pp. 335-345.

[29] B. E. Rhoades, Fixed point iterations for certain nonlinear mappings, J. Math. Anal. Appl. 183 (1994), no. 1, 118-120.

[30] K.-K. Tan and H.-K. Xu, Approximating fixed points of nonexpansive mappings by the Ishikawa iteration process, J. Math. Anal. Appl. 178 (1993), no. 2, 301-308.

[31] X. L. Weng, Fixed point iteration for local strictly pseudo-contractive mapping, Proc. Amer. Math. Soc. 113 (1991), no. 3, 727-731.

[32] H. K. Xu, Inequalities in Banach spaces with applications, Nonlinear Anal. 16 (1991), no. $12,1127-1138$.

[33] E. Zeidler, Nonlinear Functional Analysis and Its Applications. II. Monotone Operators, Springer-Verlag, Berlin, 1985.

[34] H. Zhou and Y. Jia, Approximation of fixed points of strongly pseudocontractive maps without Lipschitz assumption, Proc. Amer. Math. Soc. 125 (1997), no. 6, 17051709.

C. E. Chidume: The Abdus Salam International Centre for Theoretical Physics (ICTP), 34100 Trieste, Italy

E-mail address: chidume@ictp.trieste.it

H. Zegeye: The Abdus Salam International Centre for Theoretical Physics (ICTP), 34100 Trieste, Italy

E-mail address: habz@ictp.trieste.it 


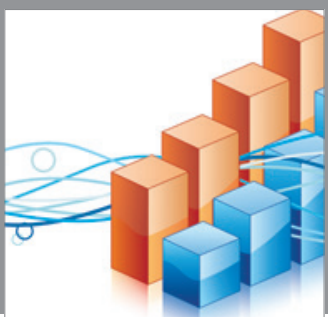

Advances in

Operations Research

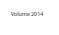

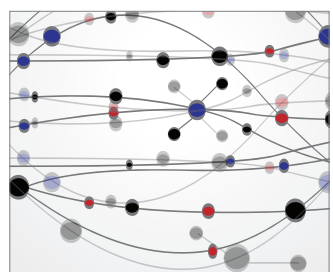

\section{The Scientific} World Journal
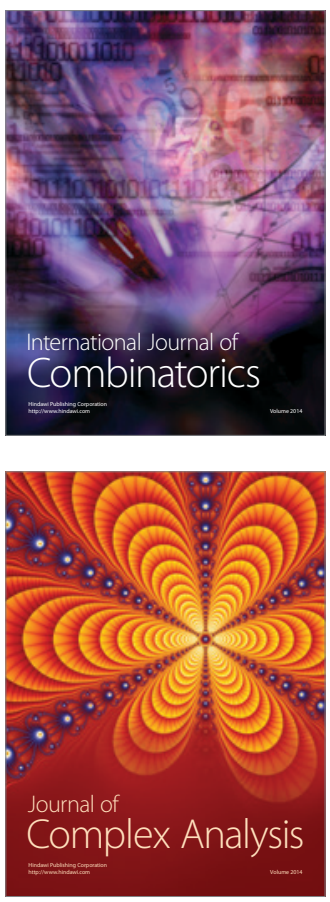

International Journal of

Mathematics and

Mathematical

Sciences
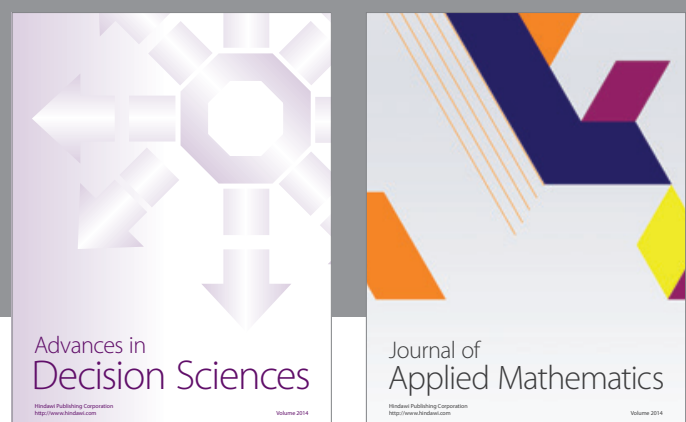

Journal of

Applied Mathematics
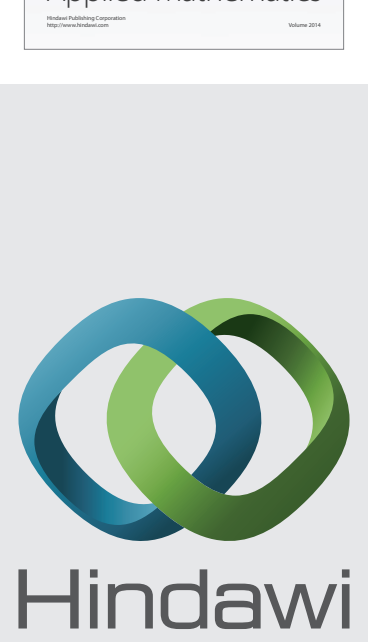

Submit your manuscripts at http://www.hindawi.com
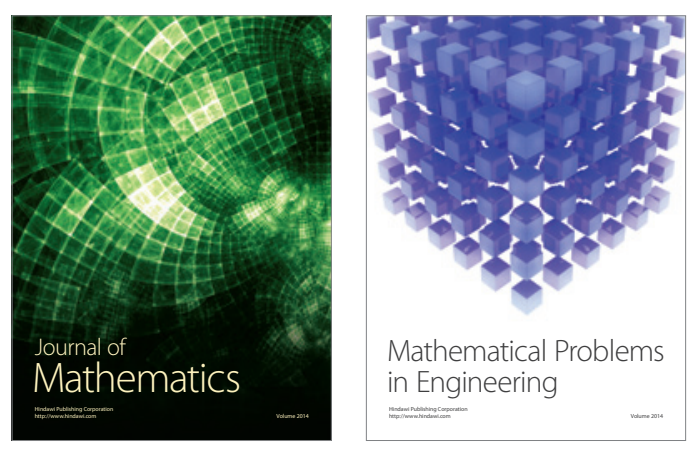

Mathematical Problems in Engineering
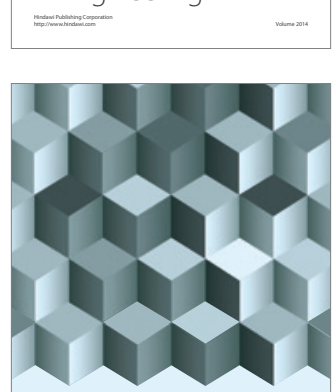

Journal of

Function Spaces
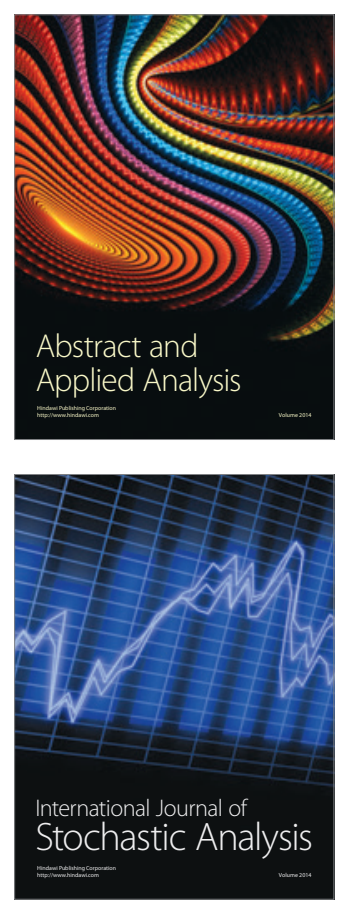

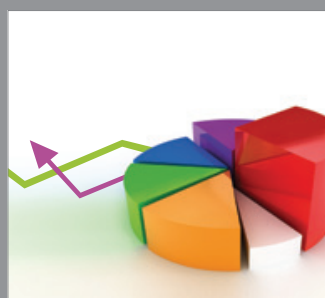

ournal of

Probability and Statistics

Promensencen
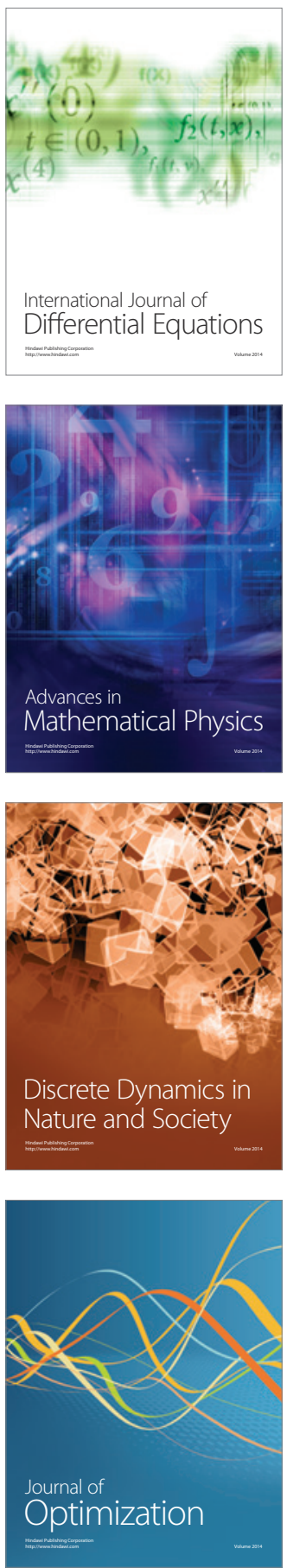